\title{
DESIGN AND SIMULATION OF MEMS BASED BIO-SENSOR FOR THE DETECTION OF CHOLERA DISEASE
}

\author{
K. Srinivasa Rao ${ }^{1}$, K. Girija Sravani ${ }^{2}$, Y.Srinivas ${ }^{3}$, J. Vijayasekhar ${ }^{4}$, G. Yugandhar $^{5}$ \\ ${ }^{1}$ Department of ECE, K L University, Vaddeswaram, Andhra Pradesh, India \\ ${ }^{2}$ Department of ECE, $K$ L University, Vaddeswaram, Andhra Pradesh, India \\ ${ }^{3}$ Department of IT, GITAM University, Hyderabad, Andhra Pradesh, India \\ ${ }^{4}$ Department of Maths, GITAM University, Hyderabad, Andhra Pradesh, India \\ ${ }^{5}$ Department of CSE, GITAM University, Hyderabad, Andhra Pradesh, India
}

\begin{abstract}
This paper focuses on biological applications of bio-sensor which can detect the presence of bacteria that causes cholera disease. This mainly concentrates on a bio sensing device which works on the principle of capacitance modulation with respect to the chemical composition of the material. The main components in the device are micro fluidic structure, functionalized sensing block, measurements system. The sensing part is the Capacitance modulation in the presence and absence of bacteria in the fluid. The capacitance value measured is now given to a chip. The chip consists of capacitor to voltage converter, signal conditioning unit. Finally, The output of chip is given to the display unit.
\end{abstract}

Keywords: Bio-Sensors, Capacitance Modulation

\section{INTRODUCTION}

The attack of a few strains of bacteria namely Vibrio Cholera on small digestive system results in cholera disease. It may have various ranges of side effects extending from none to extreme crossing over gentle range. The fine indication of this disease is major watery looseness of the bowels that will be carried over two days, including retching and muscle spasms. And the people who had affected by this disease mainly experience lack of hydration, chilly skin, wrinkling of hands and feet. The main vehicle of carrying this disease is water succeeding by nourishment contaminated with the cholera causing microscopic organisms. This infection leads to death causing disease like Hemolytic Uremic Syndrome. The percentage of fatality by this disease is 5 .

All this put together lead to the biological testing in order to identify the bacteria. And the testing with medical equipment became expensive, large crowd to work and maximum time to identify. And the same process of testing has to be done several times for better result. Subsequently this laborious work made the necessary mark to develop a hand-held and a professional platform for testing. Finally to meet this necessity we came up with a solution that is using a bio sensing device with capacitive modulation principle. This process yields a better throughput comparative to others.

\section{METHODOLOGY}

This device which is in the shape of helical is systematically attached to a walled small scale channel having fluidic nature .And it consists of parallel capacitor plates. The water which has to be tested in order to identify the existence of bacteria is made to pass through the channel. And the capacitance of water is calculated using

$$
\mathrm{C}=\left(\mathrm{K} * \mathrm{e}_{0} * \mathrm{~A}\right) / \mathrm{D}
$$

Where $\mathrm{K}$ is the dielectric constant

$\mathrm{D}$ is the separation between the plates

$\mathrm{e}_{\mathrm{o}}$ is the permittivity

$\mathrm{A}$ is the area of the plates

Normally the dielectric steady of water will be 80 .At some point we will pass the sample of new and ultra-clean water and we will calculate the quality of capacitance. Particularly, the presence of microscopic organisms can be estimated by the gradual decrement in the dielectric steady of water. The decrement will be upto 40.The main issue in our hand is to separate the Vibrio cholera bacteria from all other microscopic organisms present in the water. In order to meet this requirement we will outline the device with the estimated thickness so that bacteria just pass through it. This outlining makes easy to the device to identify the area of bacteria in the water tests and they can be removed by cleaning the water in and around the outlining. This analytical testing brought a sensitive bio-sensor which can separate the small traces of bacteria in the area of water. In order to increase the sensitivity of the device we can enhance the area of the sensor leading to the increase of capacitance worth and the accuracy.

\section{MODEL DEFINITION IN MULTIPHYSICS}

\section{TM}

The design is done by suing electrostatic and incompressible Navier strokes modes in comsol multi-physics. 
The electrostatic mode uses the definition

$$
\mathrm{Q}=\mathrm{C}^{*} \mathrm{~V}
$$

Where $\mathrm{C}$ is the capacitance

$\mathrm{V}$ is the voltage applied

$\mathrm{Q}$ is the charge developed

Here Figure 1 and Figure 2 rectangular shape model with and without bacteria. Figure 2 represents the presence of single bacteria in between the plates. The model is done with dimensions 50um X 10um representing width and channel size. Initially we have taken the water having dielectric steady of 80 and the rectangular shape is having two plates one having 10v of application while the second one is a ground. Figure 3 represents the rectangular block with electrodes. Normally the bacteria here are considered as an ellipsoid having dimensions $2 \mathrm{um} \mathrm{X} 0.65 \mathrm{um}$. The dielectric constant of ellipsoid is 40 indicating the presence of bacteria. Figure 4 and 5 correspondingly represents Micro fluidic channel structure and overview of side walls.

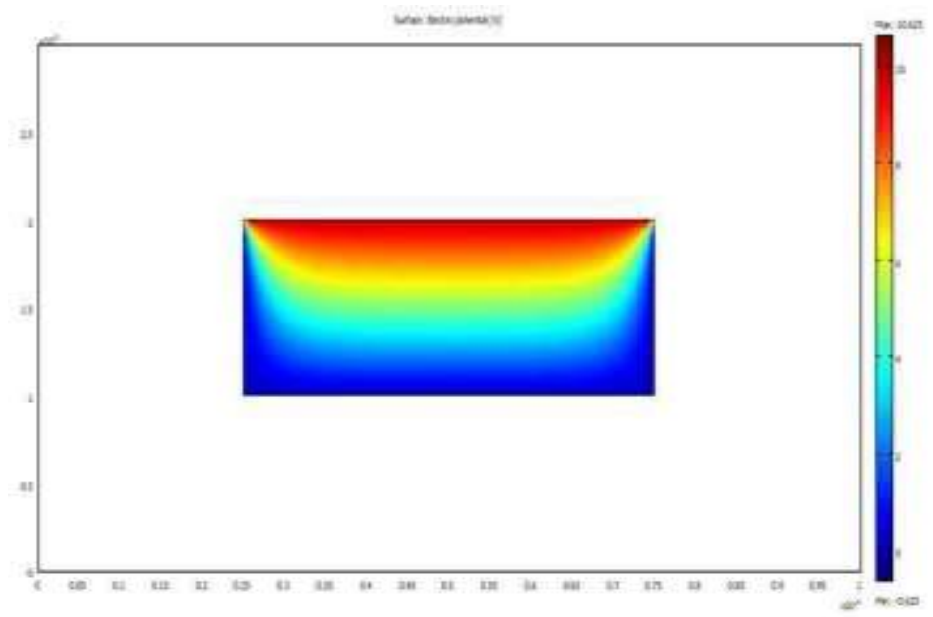

Fig 4.1: Rectangular model without bacteria

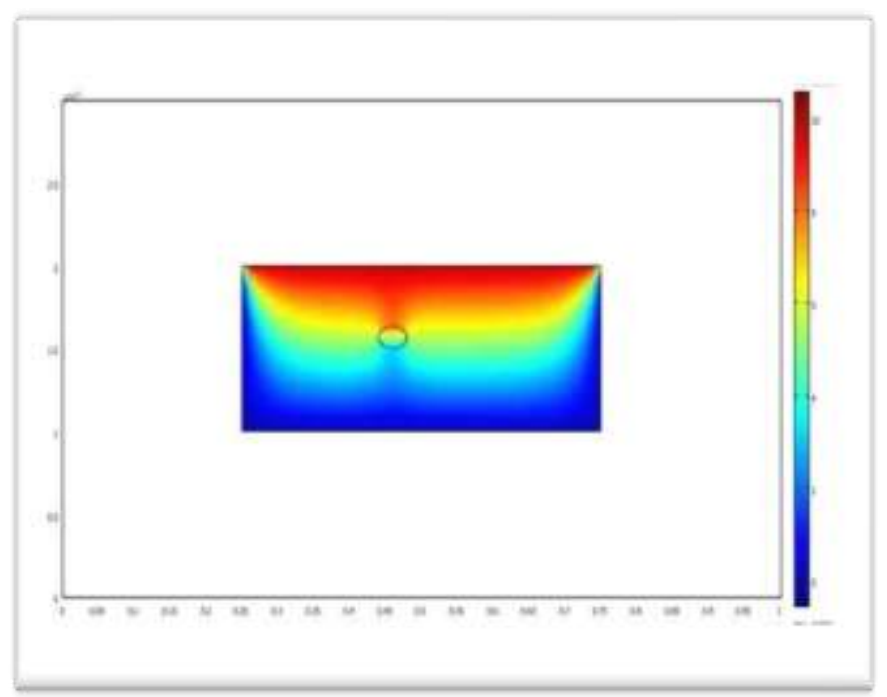

Fig 4.2: Rectangular model with bacteria

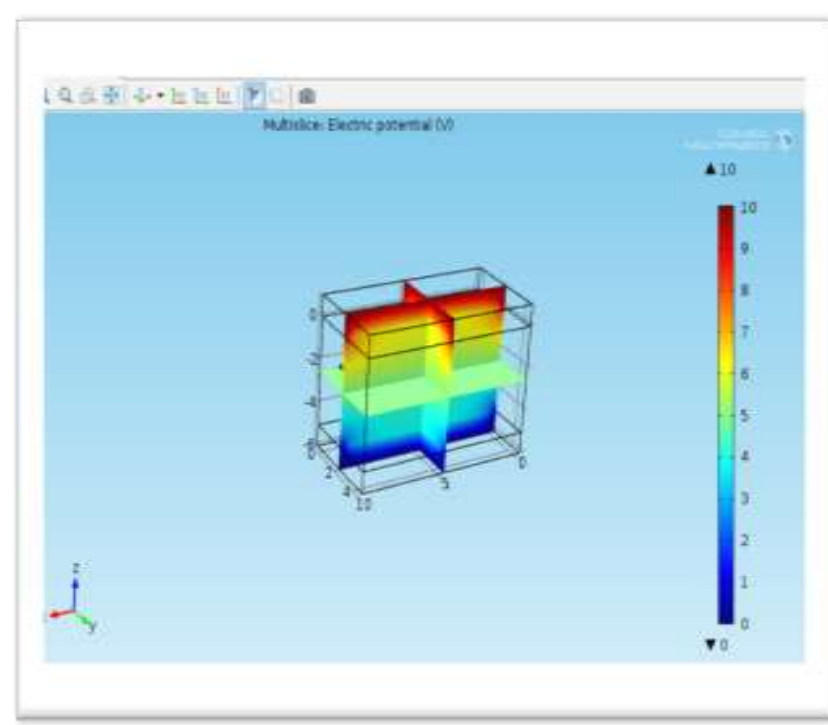

Fig 4.3: Rectangular block with electrodes

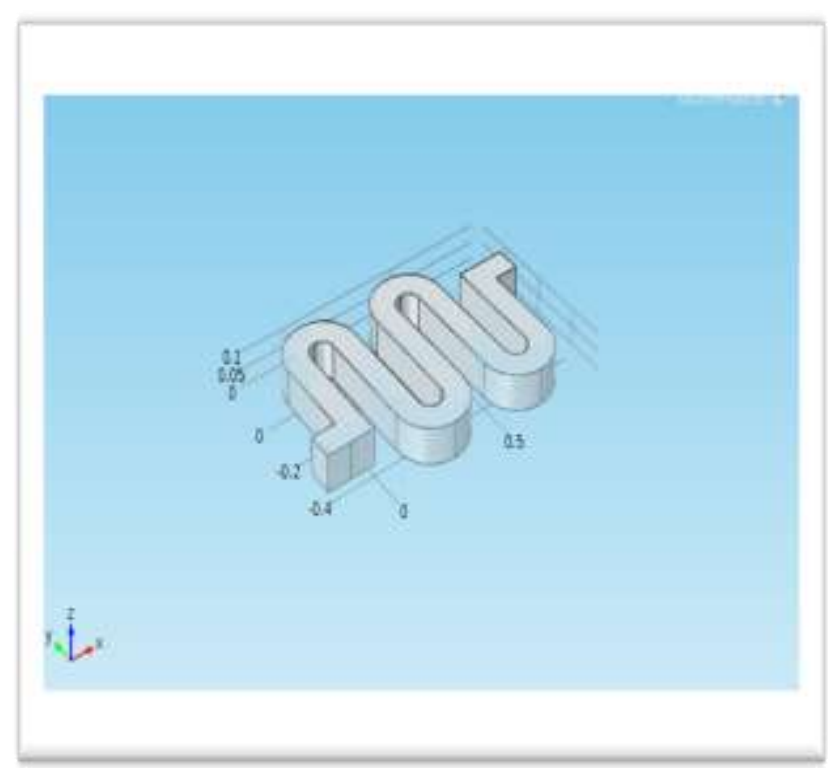

Fig 4.4 : Micro Fluidic Channel Structure

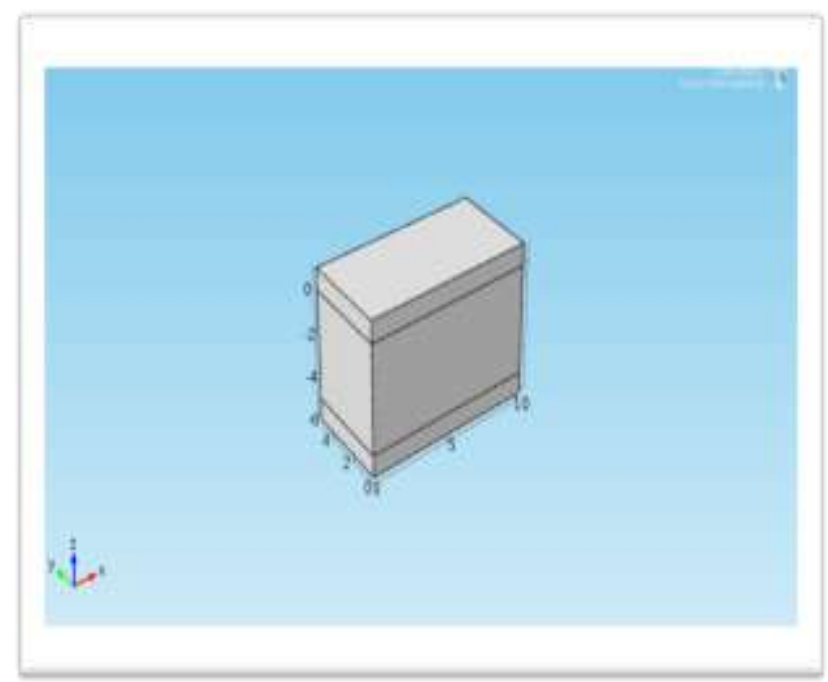

Fig 4.5: over view of side walls 


\section{ANALYSIS OF MODEL}

The sensitivity and accuracy improvement can be done by increasing the area of the sensor succeeding by the increment of capacitance.

For this model sensitivity is defined by

$$
\mathrm{S}=\left(\mathrm{C}-\mathrm{C}^{\prime}\right) / \mathrm{C}
$$

where $\mathrm{C}$ is the capacitance without bacteria

$\mathrm{C}^{\prime}$ is the capacitance with bacteria

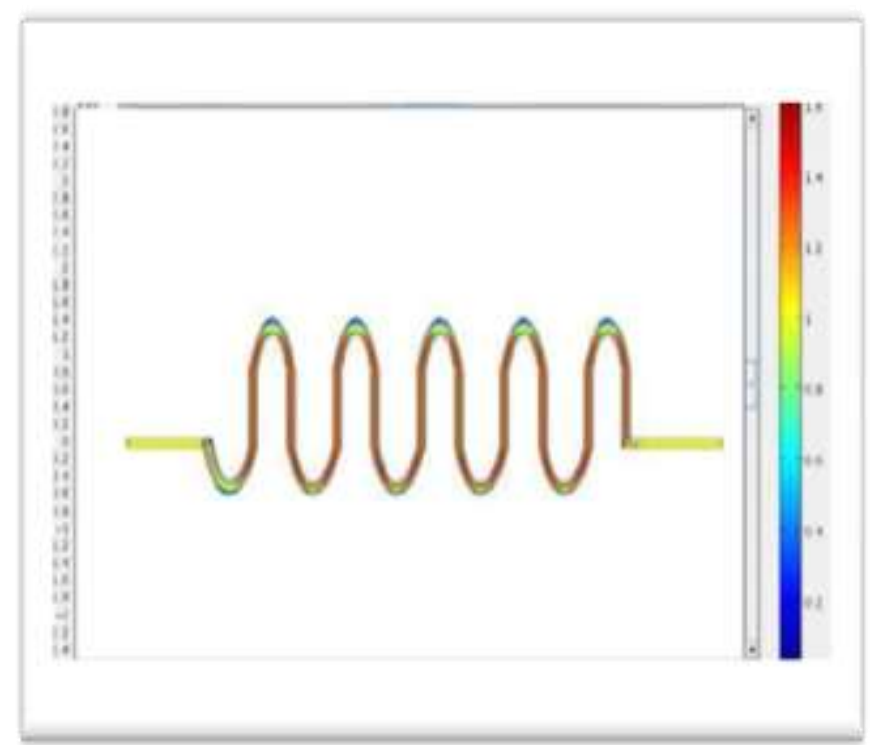

Fig 5.1: Sensor with increased overlap area without bacteria

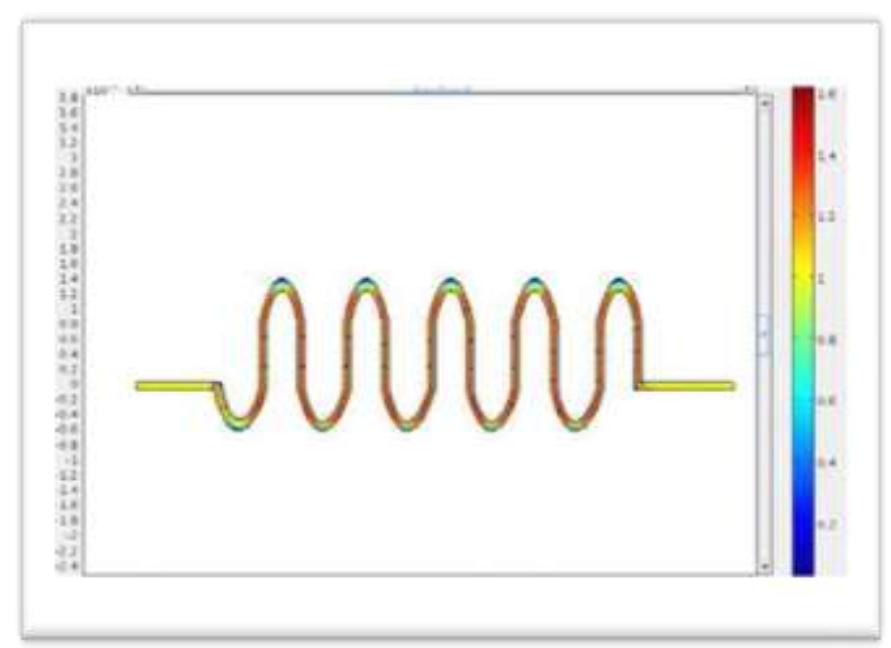

Fig 5.2: Sensor with increased overlap area and with 20 bacteria

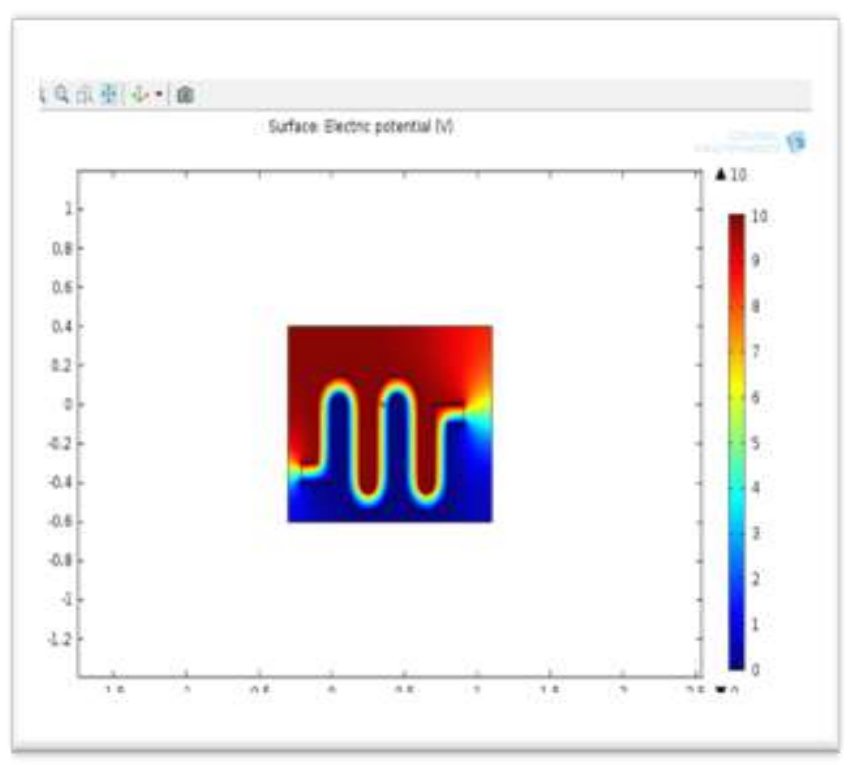

Fig 6: Fluidic channel with bacteria

\section{CONCLUSION}

The design of this particular device based on bio sensor and capacitive modulation is best for bio-sensing applications. The point where the water is passed through the channel made of capacitor plates along the dividers of the channel have one value of dielectric steady and that value changes instantaneously when the water contains microbes. And the accuracy and precision of the device is very efficient.

\section{REFERENCES}

[1] Lab on Chip for Detection of E.coli. Bacteria in Water using Capacitance modulation.- Sumeet Kailash Gupta, Corresponding author: National MEMS Design Centre

[2] Microelectrodes on a Silicon Chip for Label-Free Capacitive DNA Sensing-Carlotta Guiducci, Claudio Stagni, Alessandra Fischetti, Ubaldo Mastromatteo, Luca Benini, Senior Member, IEEE, and Bruno Riccò, Fellow, IEEE

[3] Investigation of Interconnect Capacitance Characterization Using Charge-Based Capacitance Measurement (CBCM) Technique and ThreeDimensional SimulationDennis Sylvester, Student Member, IEEE, James C. Chen, Student Member, IEEE, and Chenming Hu, Fellow, IEEE

[4] Analysis, Designing and Working Principal of Optical Fiber (OF) Biosensors- Md. Sadique Shaikh1 \& Madhuri. A. Patil. 2. 1) Dept. of Computer, Arts and Science College, Bhalod 2) Dept. of Botany, D.D.N.B College, Bhusawal

[5] Working Principle, Mechanism and Lift Effectiveness of Nano biosensors As A Food Perspective -Buket Yalcin, Semih Otles, Department of Food Engineering, Ege University of Izmir 35100 Bornova Izmir, Turkey. 